\title{
Driver mutations (JAK2V617F, MPLW515L/K or $(A L R)$, pentraxin-3 and C-reactive protein in essential thrombocythemia and polycythemia vera
}

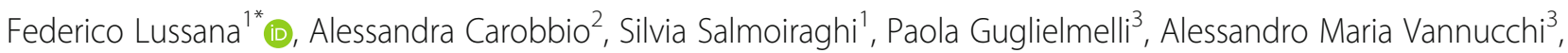
Barbara Bottazzi ${ }^{4}$, Roberto Leone ${ }^{4}$, Alberto Mantovani ${ }^{4,5}$, Tiziano Barbui ${ }^{2}$ and Alessandro Rambaldi, ${ }^{1,6}$

\begin{abstract}
Background: The driver mutations JAK2V617F, MPLW515L/K and CALR influence disease phenotype of myeloproliferative neoplasms (MPNs) and might sustain a condition of chronic inflammation. Pentraxin 3 (PTX3) and high-sensitivity C-reactive protein (hs-CRP) are inflammatory biomarkers potentially useful for refining prognostic classification of MPNs.

Methods: We evaluated 305 with essential thrombocythemia (ET) and 172 polycythemia vera (PV) patients diagnosed according to the 2016 WHO criteria and with full molecular characterization for driver mutations.

Results: PTX3 levels were significantly increased in carriers of homozygous JAK2V617F mutation compared to all the other genotypes and triple negative ET patients, while hs-CRP levels were independent of the mutational profile. The risk of haematological evolution and death from any cause was about 2- and 1.5-fold increased in individuals with high PTX-3 levels, while the thrombosis rate tended to be lower. High hs-CRP levels were associated with risk of haematological evolution, death and also major thrombosis. After sequential adjustment for potential confounders (age, gender, diagnosis and treatments) and the presence of JAK2V617F homozygous status, high hs-CRP levels remained significant for all outcomes, while JAK2V617F homozygous status as well as treatments were the factors independently accounting for adverse outcomes among patients with high PTX3 levels.

Conclusions: These results provide evidence that JAK2V617F mutation influences MPN-associated inflammation with a strong correlation between allele burden and PTX3 levels. Plasma levels of hs-CRP and PTX3 might be of prognostic value for patients with ET and PV, but their validation in future prospective studies is needed.
\end{abstract}

Keywords: Polycythemia vera, Essential thrombocythemia, Mutations, Inflammation

\section{Background}

Polycythemia vera (PV) and essential thrombocythemia (ET) are chronic myeloproliferative neoplasms (MPNs) characterized by clonal expansion of an abnormal haematopoietic progenitor cell and a clinical course that is complicated by frequent cardiovascular complications as well as increased risk of transformation to myelofibrosis (MF) or acute leukaemia (AL) $[1,2]$. The discovery of

\footnotetext{
* Correspondence: flussana@asst-pg23.it

'Hematology and Bone Marrow Transplant Unit, ASST Papa Giovanni XXIII

Bergamo, Piazza OMS, 1, 24127 Bergamo, Italy

Full list of author information is available at the end of the article
}

the Janus kinase 2 (JAK2) V617F mutation significantly improved the understanding of the biology of these disorders [3]. The subsequent identification of other acquired somatic lesions, such as JAK2 exon 12 mutation [4], mutation in the gene encoding thrombopoietin receptor (MPLW515L/K) [5] and the recently discovered mutations in the exon 9 of calreticulin $(C A L R)$ gene $[6,7]$ reinforced the central role of cytokine receptor/signal transduction lesions in promoting MPN phenotypes. In surveys of large MPN cohorts, JAK2V617F can be detected in 95\% of patients with PV and JAK2 exon12 mutation in the remaining 5\% of patients. Among ET patients, JAK2V617F 
can be detected in 60 to $65 \%$ of patients, $M P L W 515 \mathrm{~L} / \mathrm{K}$ in about $5 \%$ and CALR mutation in about 20 to $25 \%$. The understanding that an array of somatic mutations contributes to the biology of these disorders $[8,9]$ prompted new studies to address the impact of patients' mutation background on disease phenotype. These studies demonstrated that somatic mutations, by determining a more pronounced activation of platelets, leukocytes and endothelial cells $[10,11]$ and promoting an increased number of leukocytes [12] can sustain a condition of chronic inflammation, which may contribute not only to the premature atherosclerosis underlying the cardiovascular events but also to clonal evolution and second cancer [2, 13-17]. Based on these data, there is an increasing interest in two inflammatory biomarkers, belonging to the superfamily of pentraxins, such as pentraxin 3 (PTX3) and highsensitivity C-reactive protein (hs-CRP). We hypothesized that these inflammatory markers might be useful to improve prognostic classification of patients with ET and PV. In a previous paper, we showed that PTX3 and hs-CRP levels were correlated with $J A K 2$ mutation allelic burden and associated with different risks of thrombosis, although in opposite directions [18]. Furthermore, elevated hs-CRP levels were associated with shortened leukaemia-free survival (LFS) in myelofibrosis [19]. However, whether or not somatic mutations, other than JAK2V617F, influence the blood levels of PTX3 and hs-CRP is unknown and their association with the main incident relevant outcomes in ET and PV patients has not been fully explored. Accordingly, in this cross-sectional study, we examined a large cohort of patients with PV and ET.

\section{Methods}

Blood samples of 477 patients with ET and PV, diagnosed according to the 2016 World Health Organization (WHO) criteria [20], were obtained from consecutive, well-characterized patients, regularly followed in two Italian haematological centers (Bergamo and Florence). Institutional review board approval was obtained from the two participating centres in the framework of the AIRCGruppo Italiano Malattie Mieloproliferative (AGIMM) project. Blood samples were obtained at diagnosis in 172 patients (36\%) or during follow-up in 306 (64\%) (median time from diagnosis was 4.2 years, range $0.03-29.8$ years). The following previously published methods were used: real-time quantitative PCR for JAK2V617F [21] and highresolution melting analysis followed by bidirectional Sanger sequencing or next-generation sequencing for $M P L W 515 \mathrm{~L} / \mathrm{K}$ and $C A L R$ mutations [22]. In particular, evaluation of $J A K 2 \mathrm{~V} 617 \mathrm{~F}$ mutation was performed on the genomic DNA purified from granulocytes by qualitative or quantitative method. Qualitative assay was based on a allele-specific PCR starting from 100 ng DNA with a sensitivity defined as $0.5-2 \%$ [23]. Quantitative assay for the measurement of $J A K 2 \mathrm{~V} 617 \mathrm{~F}$ allele burden was performed by a quantitative real-time PCR assay, using $40 \mathrm{ng}$ DNA. All samples were analysed in triplicate, and the amount of JAK2V617F allele was calculated by comparison with serial dilutions of $J A K 2$ plasmids. The sensitivity of the quantitative method is $0.08-0.008 \%$ [21]. JAK2V617F mutation was classified as having low allele burden $(<50 \%$, heterozygous) or high allele burden ( $\geq 50 \%$, homozygous). Highsensitivity C-reactive protein (hs-CRP) was measured by a latex immunoassay (CardioPhaseHigh Sensitivity Siemens Healthcare Diagnostic Inc., Italy) and PTX3 plasma levels were measured by an in-house Sandwich ELISA as previously described [18].

Major outcomes recorded anytime during the follow-up period were major thrombosis, bleeding, evolution to MF or AL and death. Patients were treated according to current recommendations [24] and therapy included phlebotomy, hydroxyurea, pipobroman, busulfan and alpha interferon. Evolution to post-ET and post-PV MF and AL was diagnosed following the International Working Group for Myeloproliferative Neoplasms Research and Treatment and WHO criteria, respectively [20, 25].

\section{Statistical analysis}

Continuous values were expressed as medians and ranges, and nonparametric K-sample test on the equality of medians was used to test the null hypothesis that the $K$ samples were drawn from populations with the same median. Categorical data were given as counts and percentages; chi-square or Fisher exact test was used to test independence between groups, as appropriate. Boxplots were used to graph distributions across mutational groups. Statistical difference in means of hs-CRP and PTX3 values among mutations was tested considering JAK2V617F homozygous category as reference. Bonferroni correction was used to adjust the probability $(p)$ values because of the increased risk of the type I error when making multiple statistical tests. Incidence rate of outcomes of interest (major thrombosis, bleeding, haematological evolution and death) were expressed as \% patients/year. Logistic regression models were applied to estimate risk prediction of inflammatory biomarkers on outcomes. Multivariable models were evaluated unadjusted and sequentially adjusted for potential confounding factors. All tests for statistical significance were twotailed and a value of $p<0.05$ was chosen as the cut-off level for statistical significance. The statistical package STATA for Windows version 12 was used for analysis.

\section{Results}

General characteristics of the whole population

The 477 patients included 305 with ET (median age 58 years; 63\% females) and 172 with PV (median age 58 years; $47 \%$ females). Presenting features of the study 
population are shown in Table 1. Among 305 ET patients, JAK2V617F, MPLW515L/K and CALR mutations were detected in 190 (63\%), 14 (5\%) and 44 (14\%), respectively. The remaining 57 patients (18\%) were wild type for all three mutations (triple negative). The group of PV included 95 (55\%) patients with heterozygous JAK2V617F mutation, 64 (37\%) with homozygous $J A K 2 \mathrm{~V} 617 \mathrm{~F}$ mutation, 4 (2\%) with JAK2 exon 12 mutation and $9(5 \%)$ with non-mutated $J A K 2$. Median followup from diagnosis was 5.4 (range 0.01-30.7) and 6.4 (0.01-30) years in ET and PV, respectively. Aspirin was prescribed in the majority of cases and cytoreductive treatments were given in $57 \%$ and $64 \%$ of ET and PV cases, respectively. The 306 patients for whom blood samples were obtained during follow-up were receiving antiplatelet agents in the 77 and $60 \%$ and cytoreductive treatments in 65 and $68 \%$ of ET and PV cases, respectively. The majority of cytoreductive treatments used in this group of patients was hydroxyurea. In all cohorts, none of the patients was in treatment with glucocorticoids at the moment of blood sample collection, while only a minority was receiving statins as usual therapy.

Driver mutations and PTX3 and hs-CRP values distribution The median circulating plasma levels of PTX3 detected in ET and PV were similar, being respectively $4.55 \mathrm{ng} /$ $\mathrm{mL}$, range $0.58-14.95$ and $5.88 \mathrm{ng} / \mathrm{mL}$, range $0.42-23$. Similarly, the median circulating plasma levels of hsCRP were $0.83 \mathrm{mg} / \mathrm{L}$, range $0.03-7.96$ in ET and 0.87 $\mathrm{mg} / \mathrm{L}$, range $0.03-6.43$ in PV (Table 1 ). There was no correlation between hs-CRP and PTX3 both in PV and ET $(r=-0.089, p=0.29$ and $r=0.027, p=0.64$, respectively). In ET patients, hs-CRP levels were similar in triple negative and carriers of CALR, MPLW515L/K, heterozygous JAK2V617F or homozygous JAK2V617F mutations. In contrast, the circulating levels of PTX3 were significantly increased in homozygous $J A K 2 \mathrm{~V} 617 \mathrm{~F}$ mutation carriers compared to all the other genotypes (Fig. 1). Interestingly, also for PV patients, we observed significantly greater PTX3 blood levels in homozygous $J A K 2 \mathrm{~V} 617 \mathrm{~F}$ mutation carriers compared to heterozygous, while hs-CRP levels were not different by mutational status (Fig. 1). To exclude that the association between PTX3 and JAK2V617F homozygous mutation could be affected by other risk factors, we tested whether this relationship remained statistically significant after adjustment for diagnosis (PV vs. ET), gender, age and mutations (JAK2V617F homozygous vs. other mutations) in a multivariate model. This analysis confirmed a significant association only between $J A K 2 \mathrm{~V} 617 \mathrm{~F}$ homozygous status and high levels of PTX-3 only (odds ratio (OR) 1.93, 95\% confidence interval (CI) 1.44-2.59, $p<0.0001)$.

\section{Clinical outcomes according to mutational status and hs- CRP and PTX3 values}

Fifty-five major thrombotic events occurring during follow-up were registered. Thirty major thrombotic events were registered before blood sampling. The rate of thrombosis was $2.44 \%$ patients/year (95\% CI 1.35-4.41). Hemorrhagic events were observed during follow-up in a total of 43 patients (9\%); the total rate of bleeding was of 1.25 patients/year (95\% CI 0.93-1.69). A total of 33 cases of transformation to MF or AL were documented (28 to $\mathrm{MF}$ and 5 to $\mathrm{AL}$ ), accounting for an estimated incidence of haematological transformation of $0.94 \%$ patients/year (95\% CI 0.66-1.33). The rate of transformation was $0.57 \%$ patients/year (95\% CI $0.32-1.00)$ and $1.56 \%$ patients/year (95\% CI 1.01-2.41) in ET and PV patients, respectively.

Table 1 Patients' characteristics

\begin{tabular}{|c|c|c|c|}
\hline & ET & PV & Total \\
\hline$\overline{N_{1}(\%)}$ & $305(64)$ & $172(36)$ & 477 \\
\hline $\operatorname{Sex}(M / F), n(\%)$ & $112 / 193(37 / 63)$ & $91 / 81(53 / 47)$ & $203 / 274(43 / 57)$ \\
\hline $\begin{array}{l}\text { Age, years, } \\
\text { median (5th-95th percentiles) }\end{array}$ & $58.0(27.7-83.0)$ & $58.3(35.5-82.0)$ & $58.0(28.3-82.7)$ \\
\hline \multicolumn{4}{|l|}{ Mutational status, $n(\%)$} \\
\hline Triple negatives & $57(18)$ & $9(5)$ & $66(14)$ \\
\hline CALR & $44(14)$ & $0(0)$ & $44(9)$ \\
\hline MPLW515L/K & $14(5)$ & $0(0)$ & $14(3)$ \\
\hline JAK2V617F hetero & $176(58)$ & $95(55)$ & $271(57)$ \\
\hline JAK2V617F homo & $14(5)$ & $64(37)$ & $78(16)$ \\
\hline JAK2 exon 12 mutation & $0(0)$ & $4(2)$ & $4(1)$ \\
\hline $\begin{array}{l}\mathrm{Hs}-\mathrm{CRP}, \mathrm{mg} / \mathrm{L}, \\
\text { median (5th-95th percentiles) }\end{array}$ & $0.83(0.03-7.96)$ & $0.87(0.03-6.43)$ & $0.83(0.03-7.53)$ \\
\hline $\begin{array}{l}\text { PTX3, ng/mL, } \\
\text { median (5th-95th percentiles) }\end{array}$ & $4.55(0.58-14.95)$ & $5.88(0.42-22.96)$ & $4.83(0.54-18.48)$ \\
\hline
\end{tabular}

ET essential thrombocythemia, $P V$ polycythemia vera, hetero heterozygous, homo homozygous 


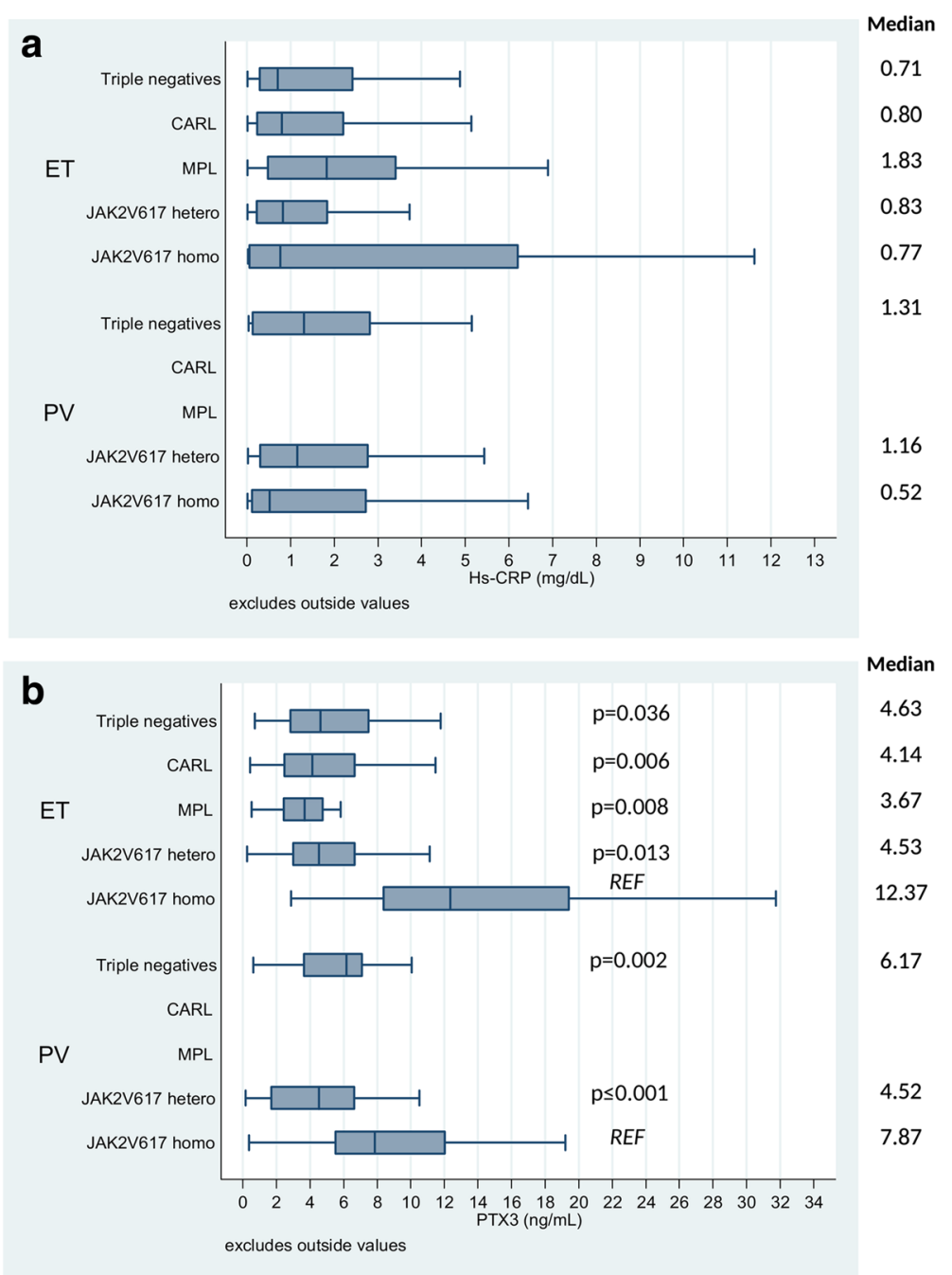

Fig. $1 \mathrm{Hs}-\mathrm{CRP}$ (a) and PTX-3 (b) values distribution according to disease and mutational status. Boxplot graphs. Analysis of multiple contrasts versus JAK2V617F homozygous as reference category (Bonferroni adjustment). Only $p$ value less than 0.05 are quoted

Among patients taking cytoreductive therapy, the incidence of haematological transformation was $1.19 \%$ patients/year (95\% CI $0.83-1.71$ ) as opposed to $0.31 \%$ (95\% CI 0.10-0.93) of patients without cytoreductive treatment. The percentage of transformation among 57 triple negative and 248 mutated ET was $0.36 \%$ patients/ year (95\% CI $0.05-2.54)$ and $0.99 \%$ patients/year $(95 \%$ CI 0.69-1.41), respectively.

During the time of this study, there were 56 deaths accounting for a mortality rate of $1.58 \%$ patients/year (95\% CI 1.22-2.06).

To test the hypothesis that different levels of hs-CRP and PTX3 are associated with different outcomes, we categorized PTX3 and hs-CRP using their respective 50th percentile of distribution in the whole cohort as cut-off point and, accordingly, we divided the study cohort in high level group for values above the 50th percentile and reference group for values below the 50th percentile. Clinical outcomes associated with high levels of PTX3 and hs-CRP are presented in Table 2. The crude relative risk for haematological evolution and death associated with PTX3 levels above the 50th percentile $(\geq 4.8 \mathrm{ng} / \mathrm{ml})$ were $1.84(95 \% \mathrm{CI}, 1.02-3.96)$ and 1.58 (95\% CI, 1.10-2.84), respectively. The multivariable analysis, after sequential adjustment for diagnosis (PV vs. ET), gender, age, treatments (cytoreductive treatments and antiplatelet agents) and mutations showed that the main factor accounting for the excess of haematological evolution and deaths was the presence of $J A K 2 \mathrm{~V} 617 \mathrm{~F}$ homozygous status, as well as the need of treatments (Table 2). In contrast, high levels of PTX3 were associated with a trend to a lower risk of thrombosis also after adjustment for JAK2V617F mutation (OR 0.57, $95 \%$ CI 0.31-1.04), suggesting a possible protective effect of PTX3. In patients with high hs-CRP levels ( $>50$ th percentile, $\geq 0.8 \mathrm{mg} / \mathrm{L}$ ) the risk of haematological evolution 
Table 2 Unadjusted and sequentially multivariable adjusted risk of principal outcomes in ET and PV patients associated to hs-CRP and PTX-3 values over their respective medians

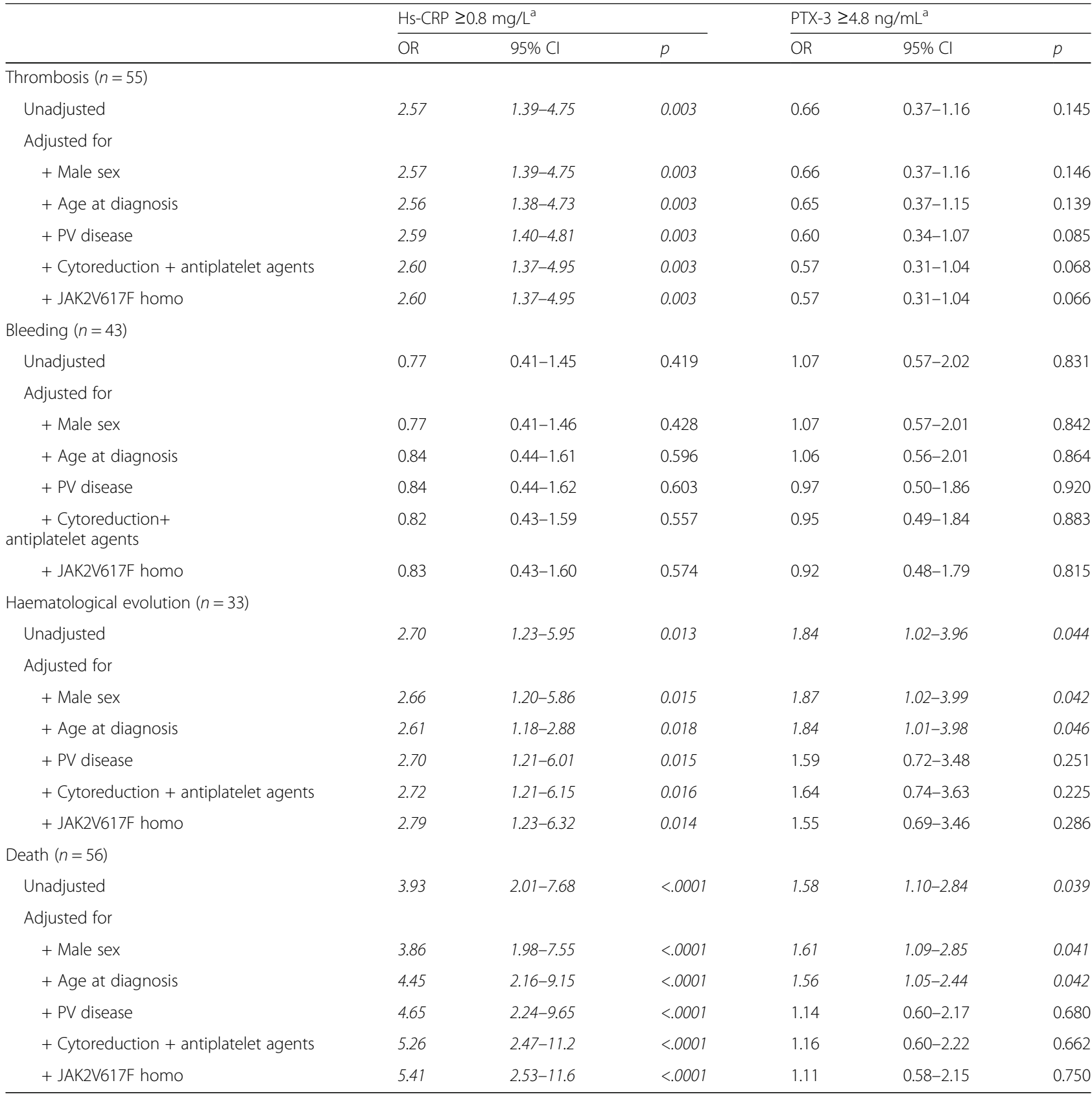

$O R$ odds ratio, $C l$ confidence interval, $p p$ value, $P V$ polycythemia vera, ASA acetylsalicylic acid

${ }^{a}$ Reference categories: hs-CRP $<0.8 \mathrm{mg} / \mathrm{L}$; PTX3 $<4.8 \mathrm{ng} / \mathrm{mL}$

The variables resulted statistically significant are reported in italicized style

and death were significantly increased compared to reference group (OR 2.70, 95\% CI 1.23-5.95 and OR 3.93, 95\% CI 2.01-7.68, respectively). Moreover, patients in the high hs-CRP group suffered also from an excess of thrombosis (OR 2.57, 95\% CI 1.39-4.75). It is worth noting that hsCRP proved an independent risk factor for adverse events in sequential multivariate analysis, also after adjustment for JAK2V617F high allele burden (Table 2). Patients in the high PTX3 or hs-CRP group showed a similar risk of bleeding compared to reference groups (OR 1.07, 95\% CI 0.572.02 and OR $0.77,95 \%$ CI 0.41-1.45, respectively) (Table 2).

\section{Discussion}

This paper conducted on a large population of ET and PV patients shows a strong association between the levels of PTX3 and JAK2V617F allele burden and also indicate that 
PTX3 and hs-CRP both reflect a condition of chronic inflammation which is associated with the disease severity.

The strong association between the levels of PTX3 and the JAK2V617F allele burden is consistent with our previous results [18] and considering the major role played by JAK2V617F mutation in the pathogenesis of inflammatory state in MPNs [10] is not surprising. Indeed, it is well established that JAK2V617F determines a more pronounced activation of platelets, leucocytes and endothelial cells [10-12] and it might induce the accumulation of reactive oxygen species in the haematopoietic stem cell compartment [26], orchestrating the construction of the inflammatory microenvironment of MPNs [27]. The association of high PTX3 levels and $J A K 2 \mathrm{~V} 617 \mathrm{~F}$ homozygous status with haematological evolution and all-cause mortality suggests that inflammation in the tumour microenvironment might contribute to the genetic instability and disease progression of MPNs $[15,28]$. Although it could be argued that only hematological transformation in AML may be due to additional mutation acquisition, the relative small number of total events prevented a formal evaluation of differences by keeping a clear distinction between the MF and AML evolution. In multivariate analysis, after adjustment for JAK2V617 allele burden, the association between PTX3 levels and adverse outcomes was no longer statistically significant, suggesting that PTX3 levels might simply reflect inflammation derived from constitutively activated blood cells, belonging to the malignant clone harbouring JAK2V617F mutation. A second relevant finding, in keeping with our previous results [18], was that the major thrombosis rate tended to be lower at the highest PTX3 levels, suggesting that PTX3 might have a protective role against the detrimental effects of inflammation in the cardiovascular risk. Preclinical studies have shown that PTX3 is released from activated leukocytes and attenuates neutrophil recruitment at sites of inflammation [29], by limiting P-selectin-dependent inflammation [30]. Moreover, PTX3 deficiency was associated with increased fibrin deposition in different models of tissue damage and a direct fibrinolytic effect of PTX3 has been described in vitro [31]. In addition, PTX3 administration resulted to be protective in a model of arterial thrombosis [32], and in carcinogenesis models, it has been demonstrated that PTX3 acts as extrinsic oncosupressor by regulating complement-mediated and macrophage-sustained tumour inflammation [33]. While it is possible that $J A K 2 \mathrm{~V} 617 \mathrm{~F}$ mutation is a potent driver of PTX3 release, the biologic and clinical correlates that can be attributed to high levels of circulating PTX3 remain to be defined and interpretations are mostly speculative. In this regard, that PTX3 might play a role in disease progression and development of fibrosis is a distinct possibility [34, 35].
In contrast to PTX3, hs-CRP levels were not associated with the JAK2V617F allele burden, supporting the hypothesis that hs-CRP and PTX3 reflect distinct aspects of the inflammatory process and that different mechanisms regulate the circulating levels of these proteins. Patients with a more pronounced chronic inflammation, as suggested by the high hs-CRP levels, exhibited an increased risk of haematological evolution and death of approximately three- and four fold, respectively, and to a lower extent also of thrombotic events compared to those in the lowest group. These findings are in keeping with previous studies in apparently healthy subjects, patients with cardiovascular disease and MPNs patients overall showing that higher levels hs-CRP are associated with an increased risk of all-cause mortality, malignant diseases and thrombotic events [18, 19, 35-39]. A striking finding of our results is that the magnitude of predictive capacity of adverse outcomes of hs-CRP seems to be significantly greater in ET and PV patients than in subjects with and without cardiovascular disease in whom the risk was only about 1.5 -fold increased in individuals with highest hs-CRP levels compared to those with lowest levels [37, 40, 41].

We acknowledge that our study has some limitations. First, PTX3 and hs-CRP were measured only once and we could not account for intra-individual variations. In this regard, future studies with prospective, repeated evaluations of the two biomarkers will be necessary to validate their predictive value for prognostic classification. Second, biomarker measurements were not performed at the time of diagnosis in many patients; thus, we cannot ascertain whether elevated PTX-3 and hsCRP may represent sensitive markers of poor prognosis since the diagnosis, or rather along with disease duration. However, this limitation does not negatively affect the validity of established correlation between high levels of PTX3 and JAK2V617F homozygous status, as well as the prognostic relevance of both biomarkers, which reflect the main hypotheses of the current study. Finally, the mechanisms by which PTX3 and hs-CRP are associated with adverse outcomes have not been addressed and our data do not allow to distinguish between the equally tenable explanations that the two biomarkers are simply a marker or rather are mechanistic determinants of patients' total inflammatory burden. On the other hand, the strength of this study is the high number of well-characterized and molecularly annotated patients that were evaluated with incident assessed outcomes.

\section{Conclusions}

The strong correlation between JAK2V617F allele burden and PTX3 levels provides further evidence to support the role of the JAK2V617F mutation as a key driver of the MPN-associated chronic inflammation. These results may 
also represent the groundwork to perform prospective studies aimed at evaluating the impact of treatments targeting simultaneously the clonal haematopoiesis (for example, with interferon-alpha2) and the accompanying inflammatory status (with agents such as JAK1/2 inhibitors, statins and histone deacetylase inhibitors) on the risk of inflammationdriven disease progression and cardiovascular events, especially in patients with high PTX3 and hs-CRP levels.

\section{Abbreviations \\ AGIMM: AIRC-Gruppo Italiano Malattie Mieloproliferative; AL: Acute leukaemia; CALR: Calreticulin; Cl: Confidence interval; ET: Essential thrombocythemia; hs-CRP: High-sensitivity C-reactive protein; LFS: Leukaemia-free survival; MF: Myelofibrosis; MPNs: Myeloproliferative neoplasms; OR: Odds ratio; PTX3: Pentraxin 3; PV: Polycythemia vera; WHO: World Health Organization}

\section{Acknowledgements}

Not applicable.

\section{Funding}

This study was funded in part by two grants from the Associazione Italiana per la Ricerca sul Cancro (AIRC, Milano) "Special Program Molecular Clinical Oncology $5 \times 1000 "$ to AGIMM (AIRC-Gruppo Italiano Malattie Mieloproliferative, http://www.progettoagimm.it) and to "INNATE IMMUNITY IN CANCER (IIC). MOLECULAR TARGETING AND CELLULAR THERAPY" project. Other fundings: FIRC - Fondazione Italiana per la Ricerca sul Cancro (AC fellowship), Cluster Alisei (MEDINTECH CTN01_00177_962865) and Fondazione Cariplo (contract no. 2015-0564) to AM, European LeukemiaNet Sixth Framework Programme LSH-2002-2.2.0-3 and AlL_-Associazione Italiana Lotta alla Leucemia AlL, sezione Paolo Belli, Bergamo.

\section{Availability of data and materials}

The datasets used and analysed during the current study available from the corresponding author on reasonable request.

\section{Authors' contributions}

FL collaborated in data interpretation, wrote the text and gave final approval before manuscript submission. AC analysed the data, collaborated in data interpretation, revised the manuscript and gave the final approval before manuscript submission. SS and RL performed the laboratory tests, revised the manuscript and gave the final approval before manuscript submission. PG, AMV, BB and AM designed the study, provided major intellectual contribution to the manuscript and gave the final approval before manuscript submission. TB and AR designed the study, supervised the data analysis, provided major intellectual contribution to the manuscript and gave the final approval before manuscript submission.

\section{Competing interests}

The authors declare that they have no competing interests.

\section{Consent for publication}

Not applicable

\section{Ethics approval and consent to partecipate}

Institutional review board approval was obtained from the two participating centres (Bergamo and Florence) in the framework of the AGIMM project (http:// www.progettoagimm.it). Samples were obtained after patients had provided written informed consent in accordance with the Helsinki Declaration.

\section{Author details}

${ }^{1}$ Hematology and Bone Marrow Transplant Unit, ASST Papa Giovanni XXIII Bergamo, Piazza OMS, 1, 24127 Bergamo, Italy. ${ }^{2}$ Research Foundation, ASST Papa Giovanni XXIII, Bergamo, Italy. ${ }^{3}$ Department of Experimental and Clinical Medicine, CRIMM, Center Research and Innovation of Myeloproliferative Neoplasms, Azienda Ospedaliera Universitaria Careggi, University of Florence, Florence, Italy. ${ }^{4}$ Humanitas Clinical and Research Center, Rozzano, Milan, Italy. ${ }^{5}$ Humanitas University, Rozzano, Milan, Italy. ${ }^{6}$ Department of Oncology and Hematology, University of Milan, Milan, Italy.
Received: 16 December 2016 Accepted: 17 February 2017

Published online: 22 February 2017

\section{References}

1. Elliott MA, Tefferi A. Thrombosis and haemorrhage in polycythaemia vera and essential thrombocythaemia. Br J Haematol. 2005;128:275-90.

2. Barbui T, Finazzi G, Falanga A. Myeloproliferative neoplasms and thrombosis. Blood. 2013;122:2176-84

3. Vainchenker W, Delhommeau F, Constantinescu SN, Bernard OA. New mutations and pathogenesis of myeloproliferative neoplasms. Blood. 2011 118:1723-35.

4. Scott LM, Tong W, Levine RL, Scott MA, Beer PA, Stratton MR, et al. JAK2 exon 12 mutations in polycythemia vera and idiopathic erythrocytosis. N Engl J Med. 2007;356:459-68

5. Pikman Y, Lee BH, Mercher T, McDowell E, Ebert BL, Gozo M, et al. MPLW515L is a novel somatic activating mutation in myelofibrosis with myeloid metaplasia. PLoS Med. 2006:3:e270.

6. Klampfl T, Gisslinger H, Harutyunyan AS, Nivarthi H, Rumi E, Milosevic JD, et al. Somatic mutations of calreticulin in myeloproliferative neoplasms. N Engl J Med. 2013;369:2379-90.

7. Nangalia J, Massie CE, Baxter EJ, Nice FL, Gundem G, Wedge DC, et al. Somatic CALR mutations in myeloproliferative neoplasms with nonmutated JAK2. N Engl J Med. 2013;369:2391-405

8. Rampal R, Al-Shahrour F, Abdel-Wahab O, Patel JP, Brunel JP, Mermel CH, et al. Integrated genomic analysis illustrates the central role of JAK-STAT pathway activation in myeloproliferative neoplasm pathogenesis. Blood. 2014;123:e123-33.

9. Klampfl T, Harutyunyan A, Berg T, Gisslinger B, Schalling M, Bagienski K, et al, Genome integrity of myeloproliferative neoplasms in chronic phase and during disease progression. Blood. 2011;118:167-76.

10. Falanga A, Marchetti M, Vignoli A, Balducci D, Russo L, Guerini V, et al. V617F JAK-2 mutation in patients with essential thrombocythemia: relation to platelet, granulocyte, and plasma hemostatic and inflammatory molecules. Exp Hematol. 2007;35:702-11.

11. Vannucchi AM, Guglielmelli P. JAK2 mutation-related disease and thrombosis. Semin Thromb Hemost. 2013;39:496-506.

12. Campbell PJ, Scott LM, Buck G, Wheatley K, East CL, Marsden JT, et al. Definition of subtypes of essential thrombocythaemia and relation to polycythaemia vera based on JAK2 V617F mutation status: a prospective study. Lancet. 2005;366:1945-53.

13. Carobbio A, Antonioli E, Guglielmelli P, Vannucchi AM, Delaini F, Guerini $V_{4}$ et al. Leukocytosis and risk stratification assessment in essential thrombocythemia. J Clin Oncol. 2008:26:2732-6.

14. Hasselbalch HC. Perspectives on chronic inflammation in essential thrombocythemia, polycythemia vera, and myelofibrosis: is chronic inflammation a trigger and driver of clonal evolution and development of accelerated atherosclerosis and second cancer? Blood. 2012;119:3219-25.

15. Hasselbalch HC. Chronic inflammation as a promotor of mutagenesis in essential thrombocythemia, polycythemia vera and myelofibrosis. A human inflammation model for cancer development? Leuk Res. 2013;37:214-20.

16. Landolfi R, Di Gennaro L, Barbui T, De Stefano V, Finazzi G, Marfisi R, et al. Leukocytosis as a major thrombotic risk factor in patients with polycythemia vera. Blood. 2007;109:2446-52.

17. Susini MC, Masala G, Antonioli E, Pieri L, Guglielmelli P, Palli D, et al. Risk of second cancers in chronic myeloproliferative neoplasms. Blood. 2012; 119:3861-218

18. Barbui T, Carobbio A, Finazzi G, Vannucchi AM, Barosi G, Antonioli E, et al. Inflammation and thrombosis in essential thrombocythemia and polycythemia vera: different role of C-reactive protein and pentraxin 3. Haematologica. 2011:96:315-8

19. Barbui T, Carobbio A, Finazzi G, Guglielmelli P, Salmoiraghi S, Rosti V, et al. Elevated C-reactive protein is associated with shortened leukemia-free survival in patients with myelofibrosis. Leukemia. 2013;27:2084-6.

20. Arber DA, Orazi A, Hasserjian R, Thiele J, Borowitz MJ, Le Beau MM, et al. The 2016 revision to the World Health Organization classification of myeloid neoplasms and acute leukemia. Blood. 2016;127:2391-405.

21. Jovanovic JV, Ivey A, Vannucchi AM, Lippert E, Oppliger Leibundgut E, Cassinat $B$, et al. Establishing optimal quantitative-polymerase chain reaction assays for routine diagnosis and tracking of minimal residual disease in JAK2-V617F-associated myeloproliferative neoplasms: a joint European 
LeukemiaNet/MPN\&MPNr-EuroNet (COST action BM0902) study. Leukemia. 2013;27:2032-9.

22. Tefferi A, Lasho TL, Finke CM, Knudson RA, Ketterling R, Hanson CH, et al. CALR vs JAK2 vs MPL-mutated or triple-negative myelofibrosis: clinical, cytogenetic and molecular comparisons. Leukemia. 2014;28:1472-7.

23. Baxter EJ, Scott LM, Campbell PJ, East C, Fourouclas N, Swanton S, et al. Acquired mutation of the tyrosine kinase JAK2 in human myeloproliferative disorders. Lancet. 2005;365:1054-61.

24. Barbui T, Barosi G, Birgegard G, Cervantes F, Finazzi G, Griesshammer M, et al. Philadelphia-negative classical myeloproliferative neoplasms: critical concepts and management recommendations from European LeukemiaNet. J Clin Oncol. 2011;29:761-70.

25. Barosi G, Mesa RA, Thiele J, Cervantes F, Campbell PJ, Verstovsek S, et al. Proposed criteria for the diagnosis of post-polycythemia vera and postessential thrombocythemia myelofibrosis: a consensus statement from the International Working Group for Myelofibrosis Research and Treatment. Leukemia. 2008;22:437-8.

26. Marty C, Lacout C, Droin N, Le Couedic JP, Ribrag V, Solary E, et al. A role for reactive oxygen species in JAK2 V617F myeloproliferative neoplasm progression. Leukemia. 2013;27:2187-95.

27. Mantovani A, Allavena P, Sica A, Balkwill F. Cancer-related inflammation. Nature. 2008:454:436-44

28. Skov V, Thomassen M, Riley CH, Jensen MK, Bjerrum OW, Kruse TA, et al. Gene expression profiling with principal component analysis depicts the biological continuum from essential thrombocythemia over polycythemia vera to myelofibrosis. Exp Hematol. 2012:40:771-80.

29. Deban L, Russo RC, Sironi M, Moalli F, Scanziani M, Zambelli V, et al. Regulation of leukocyte recruitment by the long pentraxin PTX3. Nat Immunol. 2010;11:328-34.

30. Norata GD, Marchesi P, Pulakazhi Venu VK, Pasqualini F, Anselmo A, Moalli F, et al. Deficiency of the long pentraxin PTX3 promotes vascular inflammation and atherosclerosis. Circulation. 2009;120:699-708.

31. Doni A, Musso T, Morone D, Bastone A, Zambelli V, Sironi M, et al. An acidic microenvironment sets the humoral pattern recognition molecule PTX3 in a tissue repair mode. J Exp Med. 2015;212:905-25.

32. Bonacina F, Barbieri SS, Cutuli L, Amadio P, Doni A, Sironi M, et al. Vascular pentraxin 3 controls arterial thrombosis by targeting collagen and fibrinogen induced platelets aggregation. Biochim Biophys Acta (BBA) - Mol Basis Dis. 2016;1862:1182-90.

33. Bonavita E, Gentile S, Rubino M, Maina V, Papait R, Kunderfranco P, et al. PTX3 is an extrinsic oncosuppressor regulating complement-dependent inflammation in cancer. Cell. 2015;160:700-14.

34. Pilling D, Cox N, Vakil V, Verbeek JS, Gomer RH. The long pentraxin PTX3 promotes fibrocyte differentiation. PLoS One. 2015;10, e0119709.

35. Pilling $D$, Gomer RH. Persistent lung inflammation and fibrosis in serum amyloid P component (APCs-/-) knockout mice. PLoS One. 2014;9, e93730,

36. Ballantyne CM, Hoogeveen RC, Bang H, Coresh J, Folsom AR, Chambless LE, et al. Lipoprotein-associated phospholipase A2, high-sensitivity C-reactive protein, and risk for incident ischemic stroke in middle-aged men and women in the Atherosclerosis Risk in Communities (ARIC) study. Arch Intern Med. 2005;165:2479-84.

37. Danesh J, Wheeler JG, Hirschfield GM, Eda S, Eiriksdottir G, Rumley A, et al. C-reactive protein and other circulating markers of inflammation in the prediction of coronary heart disease. N Engl J Med. 2004;350:1387-97.

38. Shah T, Casas JP, Cooper JA, Tzoulaki I, Sofat R, McCormack V, et al. Critical appraisal of CRP measurement for the prediction of coronary heart disease events: new data and systematic review of 31 prospective cohorts. Int J Epidemiol. 2009;38:217-31.

39. Kundu JK, Surh YJ. Inflammation: gearing the journey to cancer. Mutat Res. 2008;659:15-30

40. Fortmann SP, Ford E, Criqui MH, Folsom AR, Harris TB, Hong Y, et al. CDC/ AHA workshop on markers of inflammation and cardiovascular disease: application to clinical and public health practice: report from the population science discussion group. Circulation. 2004;110:e554-9.

41. Kaptoge S, Di Angelantonio E, Lowe G, Pepys MB, Thompson SG, Collins R, et al. C-reactive protein concentration and risk of coronary heart disease, stroke, and mortality: an individual participant meta-analysis. Lancet. 2010; 375:132-40.

\section{Submit your next manuscript to BioMed Central and we will help you at every step:}

- We accept pre-submission inquiries

- Our selector tool helps you to find the most relevant journal

- We provide round the clock customer support

- Convenient online submission

- Thorough peer review

- Inclusion in PubMed and all major indexing services

- Maximum visibility for your research

Submit your manuscript at www.biomedcentral.com/submit 